JOURNAL OF

SYMPLECTIC GEOMETRY

Volume 3, Number 4, 545-563, 2005

\title{
FLOER-NOVIKOV COHOMOLOGY AND SYMPLECTIC FIXED POINTS
}

\author{
KAORU ONO
}

\begin{abstract}
We introduce variants of Floer-Novikov cohomology and give a lower bound for the number of fixed points of time-one maps of timedependent symplectic flows under the non-degeneracy condition.
\end{abstract}

\section{Introduction}

In a previous paper [5], we studied an analog of Arnold's conjecture for fixed points of the time-one map of a time-dependent symplectic flow. We constructed the so-called Floer-Novikov cohomology and established the invariance under Hamiltonian deformations in a similar way to the case of Hamiltonian diffeomorphisms [1], [3], [6]. However, we restricted ourselves to the class of monotone or negatively monotone symplectic manifolds in order to compute Floer-Novikov cohomology. The purpose of this note is to give a lower bound for the rank of Floer-Novikov cohomology, which also gives a lower bound for the number of fixed points of such a symplectomorphism without assuming \pm -monotonicity of the symplectic manifold.

One of the difficulties arising in the computation is that the coefficient ring (the Novikov ring) of Floer-Novikov cohomology depends on the flux of the symplectic flow, hence we cannot directly compare Floer-Novikov cohomologies of symplectomorphisms with different fluxes. The idea in this paper is to construct the Floer-Novikov cohomology over a smaller coefficient ring. When two time-dependent flows are close to each other, we will give a variant of Floer-Novikov cohomologies over the same coefficient ring so that we can compare Floer cohomologies of different fluxes. Note that it is not enough to construct the Floer-Novikov cohomology theory over a common coefficient ring. In [2], we introduced the universal Novikov ring $\Lambda_{\text {nov }}$. The Floer-Novikov cochain complex, hence the Floer-Novikov cohomology, can be extended to objects over $\Lambda_{\text {nov }}$. Although the coefficient ring 
is the same, this construction does not directly allow us to compare the Floer-Novikov cohomologies with different fluxes.

The Floer cohomology of a symplectomorphism sufficiently $C^{1}$-close to the identity is calculated in terms of the Novikov cohomology of the flux. Let $M$ be a closed manifold. The Novikov cohomology $\operatorname{HN}^{*}(M ; a)$ is defined for $a \in \mathrm{H}^{1}(M ; \mathbf{R})$, and is a finitely generated module over the Novikov ring $\Lambda_{a}$. The function $a \mapsto \operatorname{rank}_{\Lambda_{a}} \operatorname{HN}^{*}(M ; a)$ attains the absolute minimum at generic $a$. Denote by min-nov ${ }^{p}(M)$ the minimum of $\operatorname{rank}_{\Lambda_{a}} \operatorname{HN}^{p}(M ; a)$, which we call the $p$ th minimal Novikov number. One of results in this note is the following:

Theorem 1.1. Let $\phi$ be the time-one map of a symplectic flow on $(M, \omega)$. If all fixed points of $\phi$ are non-degenerate, the number of fixed points of $\phi$ is not less than $\sum_{p}$ min-nov ${ }^{p}(M)$.

Remark 1.2. In fact, the above estimate holds for the number of fixed points, which correspond to contractible 1-periodic orbits of the timedependent symplectic flow. The proof of Theorem 1.1 is based on the Floer-Novikov cohomology theory. In general, the Floer-Novikov cohomology cannot be described in terms of the Novikov cohomology of the flux, cf. Proposition 2 in [8]. (In [8], the Floer(-Novikov) cohomology uses all components of the twisted path space (or the loop space), although we only consider the component of contractible loops in this note.)

The contents are the following. In Section 2, we briefly recall Novikov cohomology theory for closed 1-forms. In Section 3, we review Floer-Novikov cohomology theory. Sections 4 and 5 are the main body of this note, where we prove the main results.

\section{Review on Novikov cohomology}

We summarize Novikov cohomology theory for reader's convenience. Let $M$ be a closed manifold and $\eta$ a closed 1-form on $M$. We call $\eta$ a Morse 1-form, when the cross-section $\eta$ of $T^{*} M$ is transversal to the zero section, i.e., all critical points of local primitive functions for $\eta$ are non-degenerate. Define the homomorphism $I_{\eta}: \pi_{1}(M) \rightarrow \mathbf{R}$ by integrating $\eta$ along loops. We consider the covering space $\pi: \bar{M} \rightarrow M$ corresponding to ker $I_{\eta} \subset$ $\pi_{1}(M)$. The Novikov ring $\Lambda_{[\eta]}$ is the completion of the group algebra of $\Gamma=\pi_{1}(M) / \operatorname{ker} I_{\eta}$ with respect to the filtration induced by $I_{\eta}$, i.e.,

$\Lambda_{[\eta]}=\left\{\sum a_{i} g_{i} \mid a_{i} \in \mathbf{Q}, g_{i} \in \Gamma\right.$, satisfying the following finiteness condition $\}$

For each $c \in \mathbf{R}$, the set $\left\{i \mid a_{i} \neq 0, I_{\eta}\left(g_{i}\right)<c\right\}$ is finite.

Since $\pi^{*} \eta$ is exact, there exists a smooth function $\bar{f}$ such that $d \bar{f}=\pi^{*} \eta$. For each $p \in \operatorname{Zero}(\eta)$, the Morse index at $p$ is defined by the Morse index of $\bar{f}$ at $\tilde{p}$, where $\tilde{p}$ is any element in $\pi^{-1}(p)$. 
Pick a Riemannian metric $g$ on $M$ so that the gradient flow of $\bar{f}$ with respect to $\pi^{*} g$ is of Morse-Smale type. Choose also an orientation of the local stable manifold at each $p \in \operatorname{Zero}(\eta)$. Then the Novikov cochain complex $\left(\mathrm{CN}^{*}(M, \eta, g), \delta\right)$ is defined as follows.

$$
\begin{aligned}
\mathrm{CN}^{k}(M, \eta, g)= & \left\{\sum_{i} a_{i} \tilde{p}_{i} \mid a_{i} \in \mathbf{Q}, \tilde{p}_{i} \in \operatorname{Crit}(\bar{f}),\right. \\
& \text { satisfying the following conditions }\}
\end{aligned}
$$

- The index at $\tilde{p}_{i}$ is $k$ for all $i$.

- For each $c \in \mathbf{R}$, the set $\left\{i \mid a_{i} \neq 0, \bar{f}\left(\tilde{p}_{i}\right)<c\right\}$ is finite.

$$
\delta \tilde{p}=\sum_{j}\left\langle\tilde{p}, \tilde{q}_{j}\right\rangle \tilde{q}_{j}
$$

where $\tilde{q}_{j}$ runs over critical points of $\bar{f}$ of index $p+1$. Denote by $\mathcal{M}(\tilde{p}, \tilde{q} ; g)$ the moduli space of gradient flow lines, with respect to $\pi^{*} g$, joining $\tilde{p}$ to $\tilde{q}$. Since the gradient flow is of Morse-Smale type, $\mathcal{M}(\tilde{p}, \tilde{q} ; g)$ is transversal and an oriented compact 0 -dimensional manifold. Here the orientation is determined by the orientations of the local stable manifolds at $p$ and $q$. Then we define $\langle\tilde{p}, \tilde{q}\rangle$ as the signed number of points in $\mathcal{M}(\tilde{p}, \tilde{q} ; g)$. We call the image of a gradient flow line by $\pi$ a local-gradient flow line. It is easy to see that $\delta$ preserves the finiteness condition for elements in $\mathrm{CN}^{*}(M, \eta, g)$ and $\delta \circ \delta=0$. The resulting cohomology group is called the Novikov cohomology $\operatorname{HN}^{*}(M, \eta)$ for the closed 1-form $\eta$, which enjoys the following properties:

- $\operatorname{HN}^{*}(M, \eta)$ does not depend on the Riemannian metric $g$ on $M$.

- For cohomologous Morse 1-forms $\eta_{1}$ and $\eta_{2}$, the Novikov cohomologies $\mathrm{HN}^{*}\left(M, \eta_{1}\right)$ and $\mathrm{HN}^{*}\left(M, \eta_{2}\right)$ are canonically isomorphic as modules over $\Lambda_{\left[\eta_{1}\right]}=\Lambda_{\left[\eta_{2}\right]}$.

Although the Novikov cochain complex and the Novikov ring depend on the cohomology class $[\eta]$ sensitively, we have the following:

Theorem 2.1 (cf. Theorem C.1 in [5, Appendix C]). Let $\eta_{1}$ and $\eta_{2}$ be closed 1-forms such that the corresponding abelian coverings are the same, i.e., $\operatorname{ker} I_{\eta_{1}}=\operatorname{ker} I_{\eta_{2}}$. Then the ranks of the Novikov cohomologies are the same, i.e., for each $p$,

$$
\operatorname{rank}_{\Lambda_{\left[\eta_{1}\right]}} \operatorname{HN}^{p}\left(M, \eta_{1}\right)=\operatorname{rank}_{\Lambda_{\left[\eta_{2}\right]}} \operatorname{HN}^{p}\left(M, \eta_{2}\right) .
$$

Since we will use the idea of the proof, we briefly recall the argument. Denote by $\pi: \bar{M} \rightarrow M$ the covering space corresponding to ker $I_{\eta_{1}}=\operatorname{ker} I_{\eta_{2}}$. The covering transformation group is $G=\pi_{1}(M) / \operatorname{ker} I_{\eta_{1}}$. Let $f$ be a Morse function on $M$ and $g$ a Riemannian metric on $M$ such that the gradient flow of $f$ with respect to $g$ is of Morse-Smale type. Then we have the 
Morse complex $\left(\mathrm{CM}^{*}\left(\pi^{*} f\right), \delta\right)$, where the differential is defined by counting the gradient flow lines in $\bar{M}$. Note that $\mathrm{CM}^{*}\left(\pi^{*} f\right)$ is a module over the group ring $\mathbf{Q}[G]$ and $\delta$ is a $\mathbf{Q}[G]$-module homomorphism. Since the Novikov ring $\Lambda_{\eta_{1}}$ is the completion of $\mathbf{Q}[G]$ with respect to $I_{\eta_{1}}$, we get the extension $\left(\mathrm{CM}^{*}\left(\pi^{*} f\right), \delta\right) \otimes_{\mathbf{Q}[G]} \Lambda_{\eta_{1}}$. Then we find the following isomorphism:

$$
\operatorname{HN}^{*}\left(M, \eta_{1}\right) \cong \mathrm{H}^{*}\left(\left(\mathrm{CM}^{*}\left(\pi^{*} f\right), \delta\right) \otimes_{\mathbf{Q}[G]} \Lambda_{\eta_{1}}\right) .
$$

This follows from the following observation. Without changing the cohomology class of $\eta_{1}$, we may assume that $\eta_{1}$ vanishes in a neighborhood of each critical point of $f$. For a sufficiently large $N$, the local-gradient flow lines of $N \cdot d f+\eta_{1}$ with the relative index 1 are in one-to-one correspondence with the gradient flow lines of $f$ with the relative index 1 . Therefore, the Novikov complex $\left(\mathrm{CN}^{*}\left(M, N \cdot d f+\eta_{1}\right), \delta\right)$ is isomorphic to $\left(\mathrm{CM}^{*}\left(\pi^{*} f\right), \delta\right) \otimes_{\mathbf{Q}[G]} \Lambda_{\eta_{1}}$. Since $\Lambda_{\eta_{1}}$ is a field, which is flat over $\mathbf{Q}[G]$, we have

$$
\mathrm{H}^{*}\left(\left(\mathrm{CM}^{*}\left(\pi^{*} f\right), \delta\right) \otimes_{\mathbf{Q}[G]} \Lambda_{\eta_{1}}\right) \cong \mathrm{H}^{*}\left(\mathrm{CM}^{*}\left(\pi^{*} f\right), \delta\right) \otimes_{\mathbf{Q}[G]} \Lambda_{\eta_{1}} .
$$

Combining these, we find

$$
\begin{aligned}
\operatorname{rank}_{\Lambda_{\eta_{1}}} \mathrm{HN}^{*}\left(M, \eta_{1}\right) & =\operatorname{rank}_{\Lambda_{\eta_{1}}} \mathrm{H}^{*}\left(\mathrm{CM}^{*}\left(\pi^{*} f\right), \delta\right) \otimes_{\mathbf{Q}[G]} \Lambda_{\eta_{1}} \\
& =\operatorname{rank}_{\mathrm{F}(\mathbf{Q}[G])} \mathrm{H}^{*}\left(\mathrm{CM}^{*}\left(\pi^{*} f\right), \delta\right) \otimes_{\mathbf{Q}[G]} \mathrm{F}(\mathbf{Q}[G]) .
\end{aligned}
$$

Here, we denote by $\mathrm{F}(R)$ the fractional field of an integral domain $R$. Similarly, we find

$$
\operatorname{rank}_{\Lambda_{\eta_{2}}} \mathrm{HN}^{*}\left(M, \eta_{2}\right)=\operatorname{rank}_{\mathrm{F}(\mathbf{Q}[G])} \mathrm{H}^{*}\left(\mathrm{CM}^{*}\left(\pi^{*} f\right), \delta\right) \otimes_{\mathbf{Q}[G]} \mathrm{F}(\mathbf{Q}[G]) .
$$

Thus, we get Theorem 2.1.

We also proved the following:

Theorem 2.2 (cf. Theorem C.2 in [5, Appendix C]). Let $\eta_{1}$ and $\eta_{2}$ be closed 1 -forms on $M$ such that $\operatorname{ker} I_{\eta_{1}} \subset \operatorname{ker} I_{\eta_{2}}$. Then the rank of $\operatorname{HN}^{*}\left(M, \eta_{1}\right)$ is less than or equal to the rank of $\operatorname{HN}^{*}\left(M, \eta_{2}\right)$.

In particular, when $[\eta]$ is generic in $\mathrm{H}^{1}(M ; \mathbf{R})$, i.e., the corresponding covering space $\bar{M} \rightarrow M$ is the maximal abelian covering space of $M$, the rank of its Novikov cohomology is minimal.

\section{Floer-Novikov cohomology}

In this section, we summarize material on Floer-Novikov cohomology following [5]. However, we adopt a slightly different framework, i.e., a different covering space of the loop space here, see [7] for the necessary modifications.

3.1. Review on the construction of Floer-Novikov cochain complex. Let $\eta_{t}$ be a one-parameter family of closed 1-forms on $M$. Denote by $X_{\eta_{t}}$ the vector field satisfying $i\left(X_{\eta_{t}}\right) \omega=\eta_{t}$. Integrating $X_{\eta_{t}}$, we get a one-parameter family $\varphi_{t}$ of symplectomorphisms with $\varphi_{0}=i d$. We call such $\varphi_{t}$ a time-dependent symplectic flow. As in Lemma 2.1 in [5], we deform 
$\varphi_{t}$ in such a way that the cohomology class $\left[\eta_{t}\right]$ is independent of $t$ without changing the time-one map $\varphi=\varphi_{1}$. Note that the cohomology class $[\eta]=\left[\eta_{t}\right]$ is the so-called flux of $\left\{\varphi_{t}\right\}_{0 \leq t \leq 1}$. We can also adjust and extend the isotopy $\varphi_{t}$ so that $\varphi_{t+1}=\varphi_{t} \circ \varphi_{1}$ holds. In other words, we may assume that $\eta_{t}$ is 1-periodic in $t$ and represents the same cohomology class.

On the free loop space $\mathcal{L} M$ of $M$, we define a closed 1 -form $\alpha_{\left\{\varphi_{t}\right\}}$, in a formal sense, as follows.

$$
\alpha_{\left\{\varphi_{t}\right\}}(\xi)=\int_{0}^{1} \omega(\xi, \dot{\gamma}) d t+\int_{0}^{1} \eta_{t}(\xi) d t, \quad \xi \in T_{\gamma} \mathcal{L} M
$$

We take an appropriate covering space of $\mathcal{L} M$ so that the pull-back of $\alpha_{\left\{\varphi_{t}\right\}}$ becomes exact and a $\infty / 2$-dimensional analog of Morse index, i.e., the Conley-Zehnder index, of a primitive function is well defined. More precisely, we consider the covering space $\widetilde{\mathcal{L}} M$ corresponding to $K=\operatorname{ker}\left(\mathcal{I}_{\omega}+\right.$ $\left.\mathcal{I}_{\eta}\right) \cap \operatorname{ker} \mathcal{I}_{c_{1}}$, where $\mathcal{I}_{\omega}, \mathcal{I}_{\eta}$ and $\mathcal{I}_{c_{1}}$ are homomorphisms $\pi_{1}(\mathcal{L} M) \rightarrow \mathbf{R}$ defined as follows.

Let $\left\{\gamma_{\tau}\right\}$ be a loop in $\mathcal{L} M$ and $C\left(\left\{\gamma_{\tau}\right\}\right)$ the "trace" swept by a family of loops $\gamma_{\tau}$. Each loop $\gamma_{\tau}$ is parametrized by $t \in \mathbf{R} / \mathbf{Z}=S^{1}$. We set

$$
\begin{aligned}
& \mathcal{I}_{\omega}\left(\left\{\gamma_{\tau}\right\}\right)=\int_{C\left(\left\{\gamma_{\tau}\right\}\right)} \omega, \quad \mathcal{I}_{c_{1}}\left(\left\{\gamma_{\tau}\right\}\right)=\int_{C\left(\left\{\gamma_{\tau}\right\}\right)} c_{1}(M), \\
& \mathcal{I}_{\eta}\left(\left\{\gamma_{\tau}\right\}\right)=\left.\int_{0}^{1} \eta\left(\frac{d \gamma_{\tau}}{d \tau}\right)\right|_{t=0} d \tau .
\end{aligned}
$$

When we consider the component of contractible loops, the covering space $\widetilde{\mathcal{L}} M \rightarrow \mathcal{L} M$ is described in the following way. Choose a smooth function $\widetilde{h}$ on $\bar{M}$ such that $\widetilde{d h}=\pi^{*} \eta$. Consider the space of pairs $(\widetilde{\gamma}, w)$ of a loop $\widetilde{\gamma}$ in $\bar{M}$ and a disk $w: D^{2} \rightarrow M$ such that $\pi \circ \widetilde{\gamma}=\left.w\right|_{\partial D^{2}}$. We write $(\widetilde{\gamma}, w) \sim\left(\widetilde{\gamma}^{\prime}, w^{\prime}\right)$ if and only if the following conditions hold:

$$
\begin{aligned}
\pi \circ \widetilde{\gamma} & =\pi \circ \widetilde{\gamma}^{\prime} \\
\int_{D^{2}} w^{*} \omega+\widetilde{h}(\widetilde{\gamma}(0)) & =\int_{D^{2}} w^{\prime *} \omega+\widetilde{h}\left(\widetilde{\gamma}^{\prime}(0)\right)
\end{aligned}
$$

and

$$
c_{1}(M)\left[w \sharp\left(-w^{\prime}\right)\right]=0,
$$

where $w \sharp\left(-w^{\prime}\right)$ is the two-sphere obtained by gluing $w$ and $w^{\prime}$ with the reversed orientation along the boundaries. This is an equivalence relation and $\widetilde{\mathcal{L}} M$ is identified with the quotient space $\{(\widetilde{\gamma}, w)\} / \sim$. Choose a smooth function $\widetilde{H}: \mathbf{R} / \mathbf{Z} \times \bar{M} \rightarrow \mathbf{R}$ such that $d \widetilde{H}_{t}=\pi^{*} \eta_{t}$. Here $\widetilde{H}_{t}(x)=\widetilde{H}(t, x)$. Then we define the action functional on $\widetilde{\mathcal{L}} M$ by

$$
\mathcal{A}_{\widetilde{H}}([\widetilde{\gamma}, w])=\int_{D^{2}} w^{*} \omega+\int_{0}^{1} \widetilde{H}(t, \widetilde{\gamma}(t)) d t .
$$


The index of a critical point $[\widetilde{\gamma}, w]$ is given by the Conley-Zehnder index of the 1-periodic orbit with respect to the trivialization of $(\pi \circ \widetilde{\gamma})^{*} T M$, which extends to $w^{*} T M$. The Floer-Novikov cohomology for the timedependent symplectic flow $\left\{\varphi_{t}\right\}_{0 \leq t \leq 1}$ is an $\infty / 2$-dimensional analog of Morse cohomology of $\mathcal{A}_{\widetilde{H}}$. The construction of the Floer-Novikov cochain complex is similar to the case of the Floer cochain complex for periodic Hamiltonian systems. The technology in $[\mathbf{3}, \mathbf{6}]$ enables us to extend the construction in $[\mathbf{5}]$ to time-dependent symplectic flows on general closed symplectic manifolds. From now on, we assume that all 1-periodic orbits of $\varphi_{t}$ are non-degenerate.

Pick a family $J=\left\{J_{t}\right\}$ of almost complex structures compatible with $\omega$, which is 1-periodic in $t$. We set

$$
\begin{aligned}
\operatorname{CFN}^{p}\left(\left\{\varphi_{t}\right\}, J\right)= & \left\{\sum_{i} a_{i}\left[\widetilde{\gamma}_{i}, w_{i}\right] \mid a_{i} \in \mathbf{Q},\left[\widetilde{\gamma}_{i}, w_{i}\right] \in \operatorname{Crit} \mathcal{A}_{\widetilde{H}}\right. \\
& \text { satisfying the following finiteness conditions }\} .
\end{aligned}
$$

- $\left\{i \mid a_{i} \neq 0, \mathcal{A}_{\widetilde{H}}\left(\widetilde{\gamma}_{i}, w_{i}\right)<c\right\}$ is finite for any $c \in \mathbf{R}$.

- The Conley-Zehnder index $\mu\left(\left[\widetilde{\gamma}_{i}, w_{i}\right]\right)$ equals $p$.

Note that $\mathrm{CFN}^{*}\left(\left\{\varphi_{t}\right\}, J\right)$ is the completion of the $\mathbf{Q}$-vector space freely generated by the set of critical points of $\mathcal{A}_{\widetilde{H}}$ with respect to the filtration induced by $\mathcal{A}_{\widetilde{H}}$. We introduce the Novikov ring $\Lambda_{\omega, \eta}$, which depends on the cohomology classes $[\omega] \in \mathrm{H}^{2}(M ; \mathbf{R})$ and $[\eta] \in \mathrm{H}^{1}(M ; \mathbf{R})$. Write $\Gamma=\pi_{1}(\mathcal{L} M) / K$, the covering transformation group of $\widetilde{\mathcal{L}} M \rightarrow \mathcal{L} M$. The homomorphisms $\mathcal{I}_{\omega}$ and $\mathcal{I}_{\eta}$ descend to homomorphisms $\Gamma \rightarrow \mathbf{R}$. We set

$$
\begin{aligned}
& \Lambda_{\omega, \eta}=\{ \sum a_{i} g_{i} \mid a_{i} \in \mathbf{Q}, g_{i} \in \Gamma \text { satisfying the following } \\
&\text { finiteness condition }\} .
\end{aligned}
$$

- $\left\{i \mid a_{i} \neq 0,\left(\mathcal{I}_{\omega}+\mathcal{I}_{\eta}\right)\left(g_{i}\right)<c\right\}$ is finite for any $c \in \mathbf{R}$.

For each contractible 1-periodic orbit $\gamma$, pick any lift $[\widetilde{\gamma}, w] \in \widetilde{\mathcal{L}} M$. Then they generate $\operatorname{CFN}^{*}\left(\left\{\varphi_{t}\right\}, J\right)$ as a $\Lambda_{\omega, \eta}$-module. In particular, $\operatorname{CFN}^{*}\left(\left\{\varphi_{t}\right\}, J\right)$ is a finitely generated $\Lambda_{\omega, \eta}$-module. Denote by $\Lambda_{\omega, \eta}^{0}$ the subalgebra consisting of $\sum a_{i} g_{i}$ such that $\mathcal{I}_{c_{1}}\left(g_{i}\right)=0$. (In fact, $\Lambda_{\omega, \eta}^{0}$ is a field.) Note that $\operatorname{CFN}^{p}\left(\left\{\varphi_{t}\right\}, J\right)$ is finitely generated over $\Lambda_{\omega, \eta}^{0}$ for each $p$.

The coboundary operator $\delta$ is defined by counting, with signs, connecting orbits. We call $u: \mathbf{R} \times S^{1} \rightarrow M$ a connecting orbit from $\left(\widetilde{\gamma}^{-}, w^{-}\right)$to 
$\left(\widetilde{\gamma}^{+}, w^{+}\right)$if and only if $u(\tau, t)$ satisfies the following conditions.

$$
\begin{gathered}
\frac{\partial}{\partial \tau} u(\tau, t)+J_{t}(u(\tau, t))\left(\frac{\partial}{\partial t} u(\tau, t)-X_{\eta_{t}}(u(\tau, t))\right)=0, \\
\lim _{\tau \rightarrow \pm \infty} \widetilde{u}(\tau, t)=\widetilde{\gamma}^{ \pm}(t),
\end{gathered}
$$

for some lift $\widetilde{u}: \mathbf{R} \times S^{1} \rightarrow \bar{M}$ of $u$, and

$$
\left[\widetilde{\gamma}^{+}, w^{-} \sharp u\right]=\left[\widetilde{\gamma}^{+}, w^{+}\right] .
$$

The group $\mathbf{R}$ acts on the space of connecting orbits by translation in the $\tau$-variable. The quotient space can be compactified by adding stable connecting orbits as in $[\mathbf{3}]$ to get the moduli space $\mathcal{M}\left(\left[\widetilde{\gamma}^{-}, w^{-}\right],\left[\widetilde{\gamma}^{+}, w^{+}\right]\right)$of connecting orbits. We have the Kuranishi structure on $\mathcal{M}\left(\left[\widetilde{\gamma}^{-}, w^{-}\right],\left[\widetilde{\gamma}^{+}, w^{+}\right]\right)$ of dimension $\mu\left(\widetilde{\gamma}^{+}, w^{+}\right)-\mu\left(\widetilde{\gamma}^{-}, w^{-}\right)$, which we call the relative index. Using abstract multi-valued perturbation technique, we can define the "cardinality" $\left\langle\left[\widetilde{\gamma}^{-}, w^{-}\right],\left[\widetilde{\gamma}^{+}, w^{+}\right]\right\rangle \in \mathbf{Q}$ when $\mu\left(\widetilde{\gamma}^{+}, w^{+}\right)-\mu\left(\widetilde{\gamma}^{-}, w^{-}\right)=1$ and

$$
\delta[\widetilde{\gamma}, w]=\sum\left\langle[\widetilde{\gamma}, w],\left[\widetilde{\gamma}^{\prime}, w^{\prime}\right]\right\rangle\left[\widetilde{\gamma}^{\prime}, w^{\prime}\right]
$$

where $\left[\widetilde{\gamma}^{\prime}, w^{\prime}\right]$ runs over the set of critical points of $\mathcal{A}_{\widetilde{H}}$ such that $\mu\left(\widetilde{\gamma}^{\prime}, w^{\prime}\right)=$ $\mu(\widetilde{\gamma}, w)+1$. We can also show that $\delta \circ \delta=0$. The resulting cohomology $\operatorname{HFN}^{*}\left(\left\{\varphi_{t}\right\} ; J\right)$ is called the Floer-Novikov cohomology for the timedependent symplectic flow $\varphi_{t}$, which can be shown to be independent of $J$. As in the case of Hamiltonian isotopies, we get invariance under Hamiltonian deformations.

Theorem 3.1 (Theorem 4.6 in [5]). Let $\varphi_{t}$ and $\psi_{t}$ be time-dependent symplectic flows. If $\psi_{t} \circ\left(\varphi_{t}\right)^{-1}$ is a Hamiltonian isotopy, then we have a natural isomorphism as $\Lambda_{\omega, \eta}$-modules

$$
\operatorname{HFN}^{*}\left(\left\{\varphi_{t}\right\}\right) \cong \operatorname{HFN}^{*}\left(\left\{\psi_{t}\right\}\right) .
$$

In the proof, we use a $\tau$-dependent analog of the connecting orbit equation. Let $\widetilde{H}^{-}$and $\widetilde{H}^{+}$be smooth functions on $S^{1} \times \bar{M} \rightarrow \mathbf{R}$ generating $\varphi_{t}$ and $\psi_{t}$, respectively. By the hypothesis that $\psi_{t} \circ\left(\varphi_{t}\right)^{-1}$ is a Hamiltonian isotopy, there exists a function $h$ on $S^{1} \times M$ such that $\widetilde{H}^{+}=\widetilde{H}^{-}+\pi^{*} h$. Pick a smooth function $\beta(\tau)$ such that $\beta(\tau)=0$ for $\tau<<0$ and $\beta(\tau)=1$ for $\tau>>0$. The Hamiltonian vector field of $\widetilde{H}_{t}^{-}+\beta(\tau) \pi^{*} h_{t}$ on $\bar{M}$ is invariant under the covering transformations of $\bar{M} \rightarrow M$ and descends to a symplectic vector field on $M$, which we denote by $X_{\tau, t}$. Choose a family $J_{\tau, t}$ of compatible almost complex structures such that $J_{\tau, t}$ equals $J_{t}$, resp. $J_{t}^{\prime}$ for $\tau<<0$, resp. $\tau>>0$. Let $\left[\widetilde{\gamma}^{-}, w^{-}\right]$and $\left[\widetilde{\gamma}^{+}, w^{+}\right]$be critical points of $\mathcal{A}_{\widetilde{H}^{-}}$and $\mathcal{A}_{\widetilde{H}^{+}}$, 
respectively. We consider the following differential equation, which we call the cochain homomorphism equation.

$$
\begin{gathered}
\frac{\partial}{\partial \tau} u(\tau, t)+J_{\tau, t}(u(\tau, t))\left(\frac{\partial}{\partial t} u(\tau, t)-X_{\tau, t}(u(\tau, t))\right)=0, \\
\lim _{\tau \rightarrow \pm \infty} \widetilde{u}(\tau, t)=\widetilde{\gamma}^{ \pm}(t),
\end{gathered}
$$

where $\widetilde{u}: \mathbf{R} \times S^{1} \rightarrow \bar{M}$ is a lift of $u$, and

$$
\left[\widetilde{\gamma}^{+}, w^{-} \sharp u\right]=\left[\widetilde{\gamma}^{+}, w^{+}\right] \text {. }
$$

A cochain homomorphism $\operatorname{CFN}^{*}\left(\left\{\varphi_{t}\right\}, J\right) \rightarrow \operatorname{CFN}^{*}\left(\left\{\psi_{t}\right\}, J^{\prime}\right)$ is defined by "counting" the solutions of the cochain homomorphism equation.

For any solution $u$ of the cochain homomorphism equation, the following energy estimate holds under the hypothesis that $\psi_{t} \circ\left(\varphi_{t}\right)^{-1}$ is a Hamiltonian isotopy:

$$
E(u)=\int_{-\infty}^{+\infty} \int_{0}^{1}\left|\frac{\partial u}{\partial \tau}\right|^{2} d \tau d t \leq \mathcal{A}_{\widetilde{H}^{+}}\left(\left[\widetilde{\gamma}^{+}, w^{+}\right]\right)-\mathcal{A}_{\widetilde{H}^{-}}\left(\left[\widetilde{\gamma}^{-}, w^{-}\right]\right)+C,
$$

where $C$ is a constant depending on $h$ and $\beta$. Based on this estimate, the Gromov compactness holds for solutions of the equation. We use the technique in $[\mathbf{3}, \mathbf{6}]$ to define a cochain homomorphism, which induces an isomorphism between cohomologies.

3.2. Changing the flux. For a general pair $\varphi_{t}, \psi_{t}$ of time-dependent symplectic flows, we do not have a similar energy estimate. Note also that the Novikov rings are different, when the fluxes are different. Thus, even when the energy estimate holds, the cochain homomorphism may not be obtained in the same manner. For these reasons, in [5] we could compute the Floer-Novikov cohomology for time-dependent symplectic flows only when the symplectic manifold is either monotone or negatively monotone. The argument is based on an energy estimate, which is only valid for special deformations.

In Subsections 3.2 and 3.3, we study solutions of the connecting orbit equation and the cochain homomorphism equation. Let $\pi: \widetilde{M} \rightarrow M$ be a free abelian covering space such that $\pi^{*} \eta_{t}=d \widetilde{H}_{t}$ for some smooth function. Here $\widetilde{M} \rightarrow M$ is not necessarily the minimal abelian covering space $\bar{M} \rightarrow M$ as in Section 2. $\widetilde{H}: \mathbf{R} / \mathbf{Z} \times \widetilde{M} \rightarrow \mathbf{R}$. Denote by $g_{J}$ the Riemannian metric $g_{J}\left(v_{1}, v_{2}\right)=\omega\left(v_{1}, J v_{2}\right)$. We choose $\epsilon>0$ so small that the $\epsilon$-neighborhood of each 1-periodic orbit $\gamma=\pi \circ \widetilde{\gamma}$ of $\varphi_{t}$ is a tubular neighborhood of it in $M$ and

$$
\frac{1}{4} d\left(\widetilde{\gamma}(t), \widetilde{\gamma}^{\prime}(t)\right)>\epsilon
$$

for distinct 1-periodic solutions $\widetilde{\gamma}$ and $\widetilde{\gamma}^{\prime}$ in $\widetilde{M}$ and $t \in \mathbf{R} / \mathbf{Z}$. Here $d(\cdot, \cdot)$ is the Riemannian distance with respect to $\pi^{*} g_{J}$. We may also assume the 
following condition, see Lemma 5.2 in [5]. There exists $\delta_{1}>0$ such that $\left\|X_{t}(\sigma(t))-\dot{\sigma}(t)\right\|_{L^{2}}>\delta_{1}$ for a loop $\sigma$ satisfying $\max _{t} d(\sigma(t), \gamma(t))>\epsilon$ for any 1-periodic orbit $\gamma$.

We vary the flux of the time-dependent symplectic flow in the following way. Suppose that $\varphi=\varphi_{1}$ has only non-degenerate fixed points, hence only finitely many 1-periodic orbits on $M$. Denote by $\left\{X_{t}\right\}$ the family of symplectic vector fields, which generates $\left\{\varphi_{t}\right\}$. Let $\theta_{t}$ be a 1-periodic family of closed 1-forms representing the same cohomology class such that $\theta_{t}$ vanishes on the $\epsilon$-neighborhood of each contractible 1-periodic orbit of $\varphi_{t}$. Let $\left\{\phi_{t}^{\theta_{t}}\right\}$ be the symplectic flow generated by $\left\{X_{\theta_{t}}\right\}$, where $X_{\theta_{t}}$ is defined by $i\left(X_{\theta_{t}}\right) \omega=\theta_{t}$. Then we find that $\phi_{t}^{\theta_{t}} \circ \varphi_{t}$ is generated by $d \phi_{t}^{\theta_{t}}\left(X_{t}\right)+X_{\theta_{t}}$. Clearly, there exists a constant $\delta_{2}>0$ such that, if $\left\|\theta_{t}\right\|_{C^{1}}<\delta_{2}$ for each $t,\left|X_{t}-d \phi_{t}^{\theta_{t}}\left(X_{t}\right)\right|<\delta_{1} / 3$ at each point. If necessary, we choose $\delta_{2}$ small enough so that $\delta_{2}<\delta_{1} / 3$. Under this assumption, we find that $\phi_{t}^{\theta_{t}} \circ \varphi_{t}$ has the same contractible 1-periodic orbits as $\varphi_{t}$. Suppose that $\sigma(t)$ is a contractible 1-periodic orbit of $\phi_{t}^{\theta_{t}} \circ \varphi_{t}$, which is not a 1-periodic orbit of $\varphi_{t}$. Since $\phi_{t}^{\theta_{t}}$ is the identity on the $\epsilon$-neighborhood of each contractible 1-periodic orbit $\gamma(t)$ of $\varphi_{t}$, we have

$$
d(\sigma(t), \gamma(t))>\epsilon
$$

Thus we have

$\left\|d \phi_{t}^{\theta_{t}}\left(X_{t}\right)(\sigma(t))+X_{\theta_{t}}(\sigma(t))-\dot{\sigma}(t)\right\|>\left\|X_{t}(\sigma(t))-\dot{\sigma}(t)\right\|-\frac{1}{3} \delta_{1}-\delta_{2}>\frac{1}{3} \delta_{1}>0$,

which is a contradiction. Hence $\varphi_{t}$ and $\phi_{t}^{\theta_{t}} \circ \varphi_{t}$ have the same contractible 1-periodic orbits.

From now on, we assume that the cohomology class $\left[\pi^{*} \theta_{t}\right] \in \mathrm{H}^{1}(\widetilde{M} ; \mathbf{R})$ vanishes. Simply write $\delta=\delta_{2}$. Denote by $\widetilde{h}$ a smooth function on $\mathbf{R} / \mathbf{Z} \times \widetilde{M}$ such that $\widetilde{H}+\widetilde{h}$ generates the symplectic isotopy $\left\{\phi_{t}^{\theta_{t}} \circ \varphi_{t}\right\}$. In fact, $\widetilde{h}=$ $\left(\phi_{t}^{\theta_{t}}\right)^{*} \widetilde{H}+\bar{h}^{\theta_{t}}-\widetilde{H}$, where $\bar{h}^{\theta_{t}}$ is a function on $S^{1} \times \widetilde{M}$ such that $d \bar{h}_{t}^{\theta_{t}}=\pi^{*} \theta_{t}$ for each $t \in S^{1}$.

As in subsection 3.1, we consider the following cochain homomorphism equation:

$$
\frac{\partial}{\partial \tau} u(\tau, t)+J_{\tau, t}(u(\tau, t))\left(\frac{\partial}{\partial t} u(\tau, t)-X_{\tau, t}(u(\tau, t))\right)=0
$$

with

$$
X_{\tau, t}=X_{\widetilde{H}}+\beta(\tau) X_{\widetilde{h}} .
$$

Here $\beta$ is the same function as in subsection 3.1.

Lemma 3.2. (Lemma 5.4 in [5]). If the closed 1-forms $\theta_{t}$ satisfy $\left\|\theta_{t}\right\|_{C^{0}}<\delta / 3$, then we have the following energy estimate for solutions of 
the cochain homomorphism equation for the homotopy from $\widetilde{H}^{-}=\widetilde{H}$ to $\widetilde{H}^{+}=\widetilde{H}+\widetilde{h}$

$$
E(u) \leq 3\left(\mathcal{A}_{\widetilde{H}}\left(\left[\widetilde{\gamma}^{+}, w^{+}\right]\right)-\mathcal{A}_{\widetilde{H}}\left(\left[\widetilde{\gamma}^{-}, w^{-}\right]\right)\right),
$$

where $\left[\widetilde{\gamma}^{-}, w^{-}\right]$and $\left[\widetilde{\gamma}^{+}, w^{+}\right]$are critical points of $\mathcal{A}_{\widetilde{H}}$ and $\mathcal{A}_{\widetilde{H}+\widetilde{h}}$, respectively. A similar inequality holds, when the roles of $\mathcal{A}_{\widetilde{H}}$ and $\mathcal{A}_{\widetilde{H}+\widetilde{h}}$ are exchanged, i.e., the estimate also holds for the cochain homomorphism equation for the homotopy from $\widetilde{H}+\widetilde{h}$ to $\widetilde{H}$.

This lemma guarantees the energy bound for solutions of the $\tau$-dependent analog of the connecting orbit equation with prescribed initial and final conditions. We use this fact to construct a cochain homomorphism between variants of Floer-Novikov cohomologies, which we will introduce in Section 4, with slightly different fluxes.

3.3. Energy-distance inequality. It is natural to expect that the energy of a connecting orbit $u$ is roughly bounded from below by a quantity proportional to the distance between $\widetilde{\gamma}^{-}$and $\widetilde{\gamma}^{+}$in $\widetilde{M}$. More precisely, we will prove Lemma 3.4, which is a key of the construction of the Floer-Novikov cohomology over a smaller coefficient ring.

Lemma 3.3. There exists $e>0$ with the following property. Suppose that, for a connecting orbit $u$, there exist $R_{1}<R_{2}$ and distinct 1-periodic orbits $\widetilde{\gamma}_{1}$ and $\widetilde{\gamma}_{2}$ in $\widetilde{M}$ such that $\max _{t} d\left(u\left(R_{i}, t\right), \widetilde{\gamma}_{i}(t)\right) \leq \epsilon$. Then the following inequality holds:

$$
\int_{R_{1}}^{R_{2}} \int_{0}^{1}\left|\frac{\partial u}{\partial \tau}\right|^{2} d \tau d t>e .
$$

Proof. Without loss of generality, we may assume that

- $\max _{t} d\left(u\left(R_{i}, t\right), \widetilde{\gamma}_{i}(t)\right)=\epsilon$.

- For $r \in\left(R_{1}, R_{2}\right), \max _{t} d(u(r, t), \widetilde{\gamma}(t))>\epsilon$ for any 1-periodic solution $\widetilde{\gamma}$.

In the case that $R_{2}-R_{1} \leq 1$, the Cauchy-Schwarz inequality implies that

$$
\begin{aligned}
\int_{R_{1}}^{R_{2}} \int_{0}^{1}\left|\frac{\partial u}{\partial \tau}\right|^{2} d \tau d t & \geq \int_{0}^{1}\left(\int_{R_{1}}^{R_{2}}\left|\frac{\partial u}{\partial \tau}\right| d \tau\right)^{2} d t \\
& \geq\left(\min _{t} d\left(\widetilde{\gamma}_{1}(t), \widetilde{\gamma}_{2}(t)\right)-2 \epsilon\right)^{2}>4 \epsilon^{2}
\end{aligned}
$$

In the case that $R_{2}-R_{1}>1$, we show that

$$
\int_{R_{1}}^{R_{2}} \int_{0}^{1}\left|\frac{\partial u}{\partial \tau}\right|^{2} d \tau d t>\frac{\delta^{2}}{2}
$$


Then the desired inequality holds with $e=\min \left\{4 \epsilon^{2}, \delta^{2} / 2\right\}$. We suppose the contrary. Choose a real number $R$ such that $[R, R+1] \subset\left(R_{1}, R_{2}\right)$. Since

$$
\int_{R}^{R+1} \int_{0}^{1}\left|\frac{\partial u}{\partial \tau}\right|^{2} d \tau d t \leq \frac{\delta^{2}}{2}
$$

there is $r \in(R, R+1)$ such that $\left\|\frac{\partial u}{\partial t}(r, t)-X_{t}(u(r, t))\right\|_{L^{2}}<\delta$. By the choice of $\delta, t \mapsto u(r, t)$ is $\epsilon$-close to some 1-periodic solution $\widetilde{\gamma}(t)$ for some $r \in\left(R_{1}, R_{2}\right)$, i.e., $\max _{t} d(u(r, t), \widetilde{\gamma}(t)) \leq \epsilon$. This is a contradiction.

Lemma 3.4. Let $u$ be a connecting orbit from $\left[\widetilde{\gamma}^{-}, w^{-}\right]$to $\left[\widetilde{\gamma}^{+}, w^{+}\right]$. Then the following inequality holds for $\delta$ as above:

$$
E(u) \geq \frac{\delta}{2} \rho\left(\widetilde{\gamma}^{-}, \widetilde{\gamma}^{+}\right)
$$

where

$$
\rho\left(\widetilde{\gamma}^{-}, \widetilde{\gamma}^{+}\right)=\int_{0}^{1} d\left(\widetilde{\gamma}^{-}(t), \widetilde{\gamma}^{+}(t)\right) d t
$$

This is essentially Lemma 3.5 in [5], the proof of which is made more precise below.

Proof. Firstly, we note that there are finitely many real numbers $-\infty<$ $R^{1-}<R^{1+}<R^{2-}<\cdots<R^{k-}<R^{k+}<+\infty$ and 1-periodic solutions $\widetilde{\gamma}^{0}=\widetilde{\gamma}^{-}, \widetilde{\gamma}^{1} \ldots \widetilde{\gamma}^{k}=\widetilde{\gamma}^{+}$such that

- $\max _{t} d\left(\widetilde{\gamma}^{i-1}(t), u\left(R^{i-}, t\right)\right)=\max _{t} d\left(\widetilde{\gamma}^{i}(t), u\left(R^{i+}, t\right)\right)=\epsilon$ for $i=$ $1, \ldots, k$.

- $\max _{t} d(u(\tau, t), \widetilde{\gamma}(t))>\epsilon$ for $\tau \in\left(R^{i-}, R^{i+}\right)$ and any 1-periodic solution $\widetilde{\gamma}$

Note also that $k<E(u) / e$ by Lemma 3.3. In particular, the finiteness of $k$ follows from the fact that $E(u)$ is finite.

We observe that

$$
\begin{aligned}
E_{R^{i-}}^{R^{i+}}(u) & =\int_{R^{i-}}^{R^{i+}} \int_{0}^{1}\left|\frac{\partial u}{\partial \tau}\right|^{2} d \tau d t \\
& =\int_{R^{i-}}^{R^{i+}}\left(\sqrt{\int_{0}^{1}\left|\frac{\partial u}{\partial t}(\tau, t)-X_{t}(u(\tau, t))\right|^{2}} d t\right)^{2} d \tau
\end{aligned}
$$

Applying the Cauchy-Schwarz inequality, we get

$$
E_{R^{i-}}^{R^{i+}}(u) \geq \frac{1}{R^{i+}-R^{i-}}\left(\int_{R^{i-}}^{R^{i+}} \sqrt{\int_{0}^{1}\left|\frac{\partial u}{\partial t}(\tau, t)-X_{t}(u(\tau, t))\right|^{2}} d t d \tau\right)^{2} .
$$


Using the condition for $\left.u\right|_{\left[R^{i-}, R^{i+}\right] \times S^{1}}$ and the connecting orbit equation, we get

$$
E_{R^{i-}}^{R^{i+}}(u) \geq \delta \int_{R^{i-}}^{R^{i+}} \sqrt{\int_{0}^{1}\left|\frac{\partial u}{\partial \tau}\right|^{2}} d t d \tau .
$$

Once again applying the Cauchy-Schwarz inequality, we get

$$
E_{R^{i-}}^{R^{i+}}(u) \geq \delta \int_{R^{i-}}^{R^{i+}} \int_{0}^{1}\left|\frac{\partial u}{\partial \tau}\right| d t d \tau \geq \delta\left(\rho\left(\widetilde{\gamma}^{i-1}(t), \widetilde{\gamma}^{i}(t)\right)-2 \epsilon\right) .
$$

Now we show that

$$
E_{R^{i-}}^{R^{i+}}(u) \geq \frac{\delta}{2}\left(\rho\left(\widetilde{\gamma}^{i-1}(t), \widetilde{\gamma}^{i}(t)\right)\right) .
$$

When $\widetilde{\gamma}^{i-1}=\widetilde{\gamma}^{i}$, this is obvious. Otherwise, we have $\rho\left(\widetilde{\gamma}^{i-}, \widetilde{\gamma}^{i+}\right)>4 \epsilon$, hence

$$
E_{R^{i-}}^{R^{i+}}(u) \geq \delta\left(\rho\left(\widetilde{\gamma}^{i-1}(t), \widetilde{\gamma}^{i}(t)\right)-2 \epsilon\right) \geq \frac{\delta}{2} \rho\left(\widetilde{\gamma}^{i-1}(t), \widetilde{\gamma}^{i}(t)\right)
$$

Thus we get

$$
E(u) \geq \sum_{i=1}^{k} E_{R^{i-}}^{R^{i+}}(u) \geq \frac{\delta}{2} \rho\left(\widetilde{\gamma}^{-}, \widetilde{\gamma}^{+}\right) .
$$

In the line of the proof of Lemma 3.4, we find the following:

Lemma 3.5. Let $u$ be as in Lemma 3.2. Then we have

$$
\left(\mathcal{A}_{\widetilde{H}}\left(\left[\widetilde{\gamma}^{+}, w^{+}\right]\right)-\mathcal{A}_{\widetilde{H}}\left(\left[\widetilde{\gamma}^{-}, w^{-}\right]\right)\right)>\frac{\delta}{6} \rho\left(\widetilde{\gamma}^{-}, \widetilde{\gamma}^{+}\right) .
$$

Proof. Set $\ell_{\tau}(t)=u(\tau, t)$. Suppose that the loop $\ell_{\tau}$ satisfies $\max \left(\ell_{\tau}(t), \gamma(t)\right)$ $>\epsilon$ for any 1-periodic orbit $\gamma$, then we find that

$$
\sqrt{\int_{0}^{1}\left|\frac{\partial u}{\partial \tau}\right|^{2} d t} \geq \sqrt{\int_{0}^{1}\left|\frac{\partial u}{\partial t}-X_{t}(u)\right|^{2} d t}-\frac{2 \delta}{3} \geq \frac{\delta}{3} .
$$

Taking this inequality into account, we easily adapt the proof of Lemmas 3.3 and 3.4 to obtain the desired estimate.

\section{Variants of Floer-Novikov cochain complex}

In the construction of the Floer-Novikov cohomology, the abelian covering space $\bar{M} \rightarrow M$ depends on the flux $\eta$ of the time-dependent symplectic flow. To compare Floer-Novikov cohomologies with different fluxes, we need to construct a variant of Floer-Novikov cohomology in the same abelian covering space of $M$. In this section, $\widetilde{M} \rightarrow M$ denote a free abelian covering space of $M$ such that $\pi^{*} \eta$ is exact. For instance, the maximal abelian covering space of $M$. Another choice is the smallest covering space with this property as in Section 3. With the help of Lemma 3.4, we construct two variants of the Floer-Novikov cohomology as follows. 
The first construction is almost straightforward. Let $\varphi_{t}$ be a timedependent symplectic flow with 1-periodic $\eta_{t}$. Suppose that $\pi^{*} \eta_{t}$ is exact on $\widetilde{M}$. Choose $\widetilde{H}$ such that $d \widetilde{H}_{t}=\pi^{*} \eta_{t}$. Denote by $\mathcal{A}_{\widetilde{H}}$ the functional on $\widetilde{\mathcal{L}} \widetilde{M}$ consisting of the equivalence classes of $(\widetilde{\gamma}, w)$, where $\widetilde{\gamma}$ is a loop on $\widetilde{M}$ and $w$ is a bounding disk for $\pi \circ \widetilde{\gamma}$. We write $(\widetilde{\gamma}, w) \sim\left(\widetilde{\gamma}^{\prime}, w^{\prime}\right)$ if they satisfy the following conditions.

$$
\begin{aligned}
\widetilde{\gamma} & =\widetilde{\gamma}^{\prime} \\
\int_{D^{2}} w^{*} \omega & =\int_{D^{2}} w^{\prime *} \omega
\end{aligned}
$$

and

$$
c_{1}(M)\left[w \sharp\left(-w^{\prime}\right)\right]=0 .
$$

We set

$$
\operatorname{CFN}^{p}\left(\left\{\phi_{t}\right\}, J ; \widetilde{M}\right)=\left\{\sum_{i} a_{i}\left[\widetilde{\gamma}_{i}, w_{i}\right] \mid a_{i} \in \mathbf{Q},\left[\widetilde{\gamma}_{i}, w_{i}\right] \in \operatorname{Crit} \mathcal{A}_{\widetilde{H}}\right.
$$

$$
\text { satisfying the following finiteness conditions }\} \text {. }
$$

- $\left\{i \mid a_{i} \neq 0, \mathcal{A}_{\widetilde{H}}\left(\widetilde{\gamma}_{i}, w_{i}\right)<c\right\}$ is finite for any $c \in \mathbf{R}$.

- The Conley-Zehnder index $\mu\left(\left[\widetilde{\gamma}_{i}, w_{i}\right]\right)$ equals $p$.

The coboundary operator is defined by the same formula in Section 3 . By the usual compactness argument applied to the solution $\pi \circ u$ of the perturbed Cauchy-Riemann equation with values in $M$, we find that there are only finitely many connecting orbits, whose energy is bounded by a fixed constant, of relative index 1 starting from a fixed critical point $[\widetilde{\gamma}, w] \in \widetilde{\mathcal{L}} \widetilde{M}$, after the abstract multi-valued perturbation as in [3]. Therefore, the finiteness condition is preserved by the Floer-Novikov coboundary operator. (In fact, Lemma 3.4 guarantees a stronger finiteness condition as we will see below.)

Since we work with a different covering space of the loop space, the coefficient ring of this variant of the Floer-Novikov cochain complex is also different from the one in Section 3. The covering transformation group of $\widetilde{\mathcal{L}} \widetilde{M} \rightarrow$ $\mathcal{L} M$ is isomorphic to $\Pi=\left\{\pi_{1}(M) / \pi_{1}(\widetilde{M})\right\} \oplus\left\{\pi_{2}(M) /\left(\operatorname{ker} \mathcal{I}_{\omega} \cap \operatorname{ker} \mathcal{I}_{c_{1}}\right)\right\}$. Hence the coefficient ring is the completion $\widetilde{\Lambda}_{\omega, \eta}=\Lambda_{\omega, \eta, \widetilde{M} \rightarrow M}$ of the group algebra of $\Pi$ with respect to $I_{\eta}+\mathcal{I}_{\omega}$.

Definition 4.1. We call the resulting cohomology group $\operatorname{HFN}^{*}\left(\left\{\varphi_{t}\right\}, J ; \widetilde{M}\right)$ the Floer-Novikov cohomology associated to the covering $\widetilde{M} \rightarrow M$, which is a module over $\widetilde{\Lambda}_{\omega, \eta}$.

Remark 4.2. If $[\eta] \in \operatorname{ker}^{1}(M ; \mathbf{R}) \rightarrow \mathrm{H}^{1}(\widetilde{M} ; \mathbf{R})$ is in a general position, $\operatorname{ker}\left(I_{\eta}+\mathcal{I}_{\omega}\right)=\operatorname{ker} I_{\eta} \cap \mathcal{I}_{\omega}$ and $\widetilde{M}$ coincides with $\bar{M}$ in Section 3, i.e., the 
smallest abelian covering space such that $\pi^{*} \eta$ is exact. In such a case, $\widetilde{\Lambda}_{\omega, \eta}$ coincides with $\Lambda_{\omega, \eta}$. Without such a genericity assumption on $\eta$, they need not be isomorphic and the degree zero part of $\widetilde{\Lambda}_{\omega, \eta}$ is not necessarily a principal ideal domain. But it is Noetherian.

Now we give a second variant of the Floer-Novikov cohomology. Let $U$ be a sufficiently small neighborhood of the origin in $\operatorname{ker}\left\{\pi^{*}: \mathrm{H}^{1}(M ; \mathbf{R}) \rightarrow\right.$ $\left.\mathrm{H}^{1}(\widetilde{M} ; \mathbf{R})\right\}$, which will be specified later. We consider the finiteness condition with respect to $I_{\eta+\theta}+\mathcal{I}_{\omega}$ for all $[\theta] \in U$. Namely we set

$$
\begin{aligned}
\widehat{\Lambda}_{\omega, \eta, U}= & \left\{\sum a_{i} g_{i} \mid a_{i} \in \mathbf{Q}, g_{i} \in \Pi\right. \text { satisfying the following } \\
& \text { finiteness condition }\}
\end{aligned}
$$

- $\left\{i \mid a_{i} \neq 0,\left(I_{\eta+\theta}+\mathcal{I}_{\omega}\right)\left(g_{i}\right)<c\right\}$ is finite for any $c \in \mathbf{R}$ and any $[\theta] \in U$.

Similarly we define the Floer-Novikov cochain complex as follows. Pick a function $\widetilde{h}^{\theta}: \widetilde{M} \rightarrow \mathbf{R}$ such that $d \widetilde{h}^{\theta}=\pi^{*} \theta$.

$$
\begin{aligned}
\widehat{\mathrm{CFN}}_{U}^{p}\left(\left\{\varphi_{t}\right\}, J\right)= & \left\{\sum a_{i}\left[\widetilde{\gamma}_{i}, w_{i}\right] \mid a_{i} \in \mathbf{Q},\left[\widetilde{\gamma}_{i}, w_{i}\right] \in \operatorname{Crit} \mathcal{A}_{\widetilde{H}}\right. \\
& \text { satisfying the following conditions }\} .
\end{aligned}
$$

- $\left\{i \mid a_{i} \neq 0, \mathcal{A}_{\widetilde{H}+\widetilde{h}^{\theta}}\left(\widetilde{\gamma}_{i}, w_{i}\right)<c\right\}$ is finite for any $c \in \mathbf{R}$ and any $[\theta] \in U$.

- The Conley-Zehnder index $\mu\left(\left[\widetilde{\gamma}_{i}, w_{i}\right]\right)$ equals $p$.

Remark 4.3. Let $\Theta=\left\{\theta_{t}\right\}$ be a family of closed 1-forms representing the cohomology class $[\theta]$ and $\widetilde{h}^{\Theta}$ a smooth function on $S^{1} \times \widetilde{M}$ such that $\widetilde{H}+\widetilde{h}^{\Theta}$ generates $\left\{\phi_{t}^{\theta_{t}} \circ \varphi_{t}\right\}$. Then the finiteness condition with respect to $\mathcal{A}_{\widetilde{H}+\widetilde{h}^{\theta}}$ is equivalent to the one with respect to $\mathcal{A}_{\widetilde{H}+\widetilde{h}^{\Theta}}$. In particular, the first condition is independent of the choice of the primitive function $\widetilde{h}^{\theta}$.

Let $u$ be a connecting orbit from $\left[\widetilde{\gamma}^{-}, w^{-}\right]$to $\left[\widetilde{\gamma}^{+}, w^{+}\right]$. By Lemma 3.4, we have

$$
\begin{aligned}
\left|\int_{0}^{1} \widetilde{h}^{\theta}\left(\widetilde{\gamma}^{+}(t)\right) d t-\int_{0}^{1} \widetilde{h}^{\theta}\left(\widetilde{\gamma}^{-}(t)\right) d t\right| & <\|\theta\|_{C^{0}} \rho\left(\widetilde{\gamma}^{+}, \widetilde{\gamma}^{+}\right) \\
& <2\|\theta\|_{C^{0}} \frac{E(u)}{\delta}
\end{aligned}
$$

for $\theta$ as in Section 3.2. Recall that $E(u)=\mathcal{A}_{\widetilde{H}}\left(\widetilde{\gamma}^{+}, w^{+}\right)-\mathcal{A}_{\widetilde{H}}\left(\widetilde{\gamma}^{-}, w^{-}\right)$. Thus, for a sufficiently small $\theta$, say $\|\theta\|_{C^{0}}<\delta / 4$, we find that $\mathcal{A}_{\widetilde{H}+\widetilde{h}^{\theta}}\left(\widetilde{\gamma}^{+}, w^{+}\right)>$ $\mathcal{A}_{\widetilde{H}+\widetilde{h}^{\theta}}\left(\widetilde{\gamma}^{-}, w^{-}\right)$. Hence the filtration by $\mathcal{A}_{\widetilde{H}+\widetilde{h}^{\theta}}$ is preserved under the Floer-Novikov coboundary operator $\delta$. This implies that the coboundary operator $\delta$ preserves the finiteness condition for $\widehat{\operatorname{CFN}}_{U}^{*}\left(\left\{\varphi_{t}\right\}, J\right)$, if $U$ is a sufficiently small neighborhood of the origin, say the $\delta / 4$-neighborhood of the origin. (We will choose a smaller neighborhood later.) In this way, 
we get a smaller Floer-Novikov cochain complex $\left(\widehat{\mathrm{CFN}}_{U}^{*}\left(\left\{\varphi_{t}\right\}, J\right), \delta\right)$ as a subcomplex of $\left(\mathrm{CFN}^{*}\left(\left\{\varphi_{t}\right\}, J ; \widetilde{M}\right), \delta\right)$.

Definition 4.4. We denote the resulting cohomology group by $\widehat{\operatorname{HFN}}_{U}^{*}\left(\varphi_{t}, J\right)$.

We set

$$
U=\left\{[\theta] \in \mathrm{H}^{1}(M ; \mathbf{R}) \mid\|\theta\|_{C^{0}}<\frac{\delta}{10}\right\} \cap \operatorname{ker}\left\{\pi^{*}: \mathrm{H}^{1}(M ; \mathbf{R}) \rightarrow \mathrm{H}^{1}(\widetilde{M} ; \mathbf{R})\right\} .
$$

Let $\Theta=\left\{\theta_{t}\right\}$ be a 1-periodic family of closed 1-forms representing $c \in U$ such that all $\theta_{t}$ vanish in a neighborhood of any contractible 1-periodic orbit of $\phi_{t}$. Write $U^{\prime}=U-c=\{a-c \mid a \in U\}$. Note that $\widehat{\Lambda}_{\omega, \eta, U}=\widehat{\Lambda}_{\omega, \eta+c, U^{\prime}}$. The following proposition can be shown by repeating the argument above.

Proposition 4.5. The Floer-Novikov coboundary operator preserves the $\widehat{\Lambda}_{\omega, \eta+c, U^{\prime}}$-module $\widehat{\mathrm{CFN}}_{U^{\prime}}^{*}\left(\left\{\phi_{t}^{\theta_{t}} \circ \varphi_{t}\right\}, J\right)$.

Now we compare the smaller Floer-Novikov cohomologies for $\varphi_{t}$ and $\phi_{t}^{\theta_{t}} \circ \varphi_{t}$.

Theorem 4.6. Let $\theta_{t}$ be a 1-periodic family of closed 1-forms as above. The smaller Floer-Novikov cohomologies $\widehat{\operatorname{HFN}}_{U}^{*}\left(\left\{\varphi_{t}\right\}, J\right)$ and $\widehat{\mathrm{HFN}}_{U^{\prime}}^{*}\left(\left\{\phi_{t}^{\theta_{t}}\right.\right.$ 。 $\left.\left.\varphi_{t}\right\}, J\right)$ are isomorphic as $\widehat{\Lambda}_{\omega, \eta, U}$-modules.

Proof. We may assume that $\theta_{t}$ is a 1-periodic family of sufficiently small closed 1-forms as in Section 3. The cochain homomorphism between $\widehat{\mathrm{CFN}}_{U}^{*}\left(\left\{\varphi_{t}\right\}, J\right)$ and $\widehat{\mathrm{CFN}}_{U^{\prime}}^{*}\left(\left\{\phi_{t}^{\theta_{t}} \circ \varphi_{t}\right\}, J\right)$ is defined by counting solutions of the $\tau$-dependent analog of the connecting orbit equation. Lemma 3.2 gives the energy bound for solutions with prescribed end points. Note that

$$
\left|\int_{0}^{1} \widetilde{h}_{t}^{\theta}\left(\widetilde{\gamma}^{+}(t)\right) d t-\int_{0}^{1} \widetilde{h}_{t}^{\theta}\left(\widetilde{\gamma}^{-}(t)\right) d t\right|<\|\theta\|_{C^{0}} \rho\left(\widetilde{\gamma}^{-}, \widetilde{\gamma}^{+}\right) .
$$

Lemma 3.5 implies that

$$
\rho\left(\widetilde{\gamma}^{-}, \widetilde{\gamma}^{+}\right)<\frac{6}{\delta}\left(\mathcal{A}_{\widetilde{H}}\left(\left[\widetilde{\gamma}^{+}, w^{+}\right]\right)-\mathcal{A}_{\widetilde{H}}\left(\left[\widetilde{\gamma}^{-}, w^{-}\right]\right)\right)
$$

for a solution $u$ of the cochain homomorphism equation from $\left[\widetilde{\gamma}^{-}, w^{-}\right]$to $\left[\widetilde{\gamma}^{+}, w^{+}\right]$. Thus we find that

$$
\mathcal{A}_{\widetilde{H}+\widetilde{h}^{\theta}}\left(\left[\widetilde{\gamma}^{+}, w^{+}\right]\right)>\mathcal{A}_{\widetilde{H}+\widetilde{h}^{\theta}}\left(\left[\widetilde{\gamma}^{-}, w^{-}\right]\right)
$$

for $\theta \in U$. Hence, by counting the number of solutions of the cochain homomorphism equation, we can assign an element in $\widehat{\mathrm{CFN}}_{U^{\prime}}^{*}\left(\left\{\phi_{t}^{\theta_{t}} \circ \varphi_{t}\right\}, J\right)$ to each $\left[\widetilde{\gamma}^{-}, w^{-}\right] \in \widehat{\mathrm{CFN}}_{U}^{*}\left(\left\{\varphi_{t}\right\}, J\right)$. We also see that this mapping extends to a homomorphism from $\widehat{\mathrm{CFN}}_{U}^{*}\left(\left\{\varphi_{t}\right\}, J\right)$ to $\widehat{\mathrm{CFN}}_{U^{\prime}}^{*}\left(\left\{\phi_{t}^{\theta_{t}} \circ \varphi_{t}\right\}, J\right)$.

It is easy to see that the cochain homomorphism obtained above is a $\widehat{\Lambda}_{\omega, \eta, U}$-module homomorphism. Then the rest of the standard argument in 
Floer theory goes through in a similar way and this cochain homomorphism induces an isomorphism between cohomology modules.

Let $M$ be a module over an integral domain $R$, we write $\operatorname{rank}_{R} M=$ $\operatorname{dim}_{F(R)} M \otimes_{R} F(R)$. Since the Floer-Novikov cochain complex (CFN* $\left.\left(\left\{\varphi_{t}\right\}, J\right), \delta ; \widetilde{M}\right)$ associated to the covering space $\widetilde{M} \rightarrow M$ is obtained from the smaller Floer-Novikov cochain complex $\left(\widehat{\mathrm{CFN}}_{U}^{*}\left(\left\{\varphi_{t}\right\}, J\right), \delta\right)$ by extending the coefficient ring, we have

$$
\begin{aligned}
\operatorname{rank}_{\widetilde{\Lambda}_{\omega, \eta}} \operatorname{HFN}^{*}\left(\left\{\varphi_{t}\right\}, J ; \widetilde{M}\right)= & \operatorname{dim}_{\mathrm{F}\left(\widetilde{\Lambda}_{\omega, \eta}\right)} \operatorname{HFN}^{*}\left(\left\{\varphi_{t}\right\}, J ; \widetilde{M}\right) \otimes_{\widetilde{\Lambda}_{\omega, \eta}} \mathrm{F}\left(\widetilde{\Lambda}_{\omega, \eta}\right) \\
= & \operatorname{dim}_{\mathrm{F}\left(\widetilde{\Lambda}_{\omega, \eta}\right)} \mathrm{H}^{*}\left(\left(\mathrm{CFN}^{*}\left(\left\{\varphi_{t}\right\}, J\right), \delta ; \widetilde{M}\right) \otimes_{\widetilde{\Lambda}_{\omega, \eta}}\right. \\
& \left.\mathrm{F}\left(\widetilde{\Lambda}_{\omega, \eta}\right)\right) \\
= & \operatorname{dim}_{\mathrm{F}\left(\widetilde{\Lambda}_{\omega, \eta}\right)} \mathrm{H}^{*}\left(\widehat{\mathrm{CFN}}_{U}^{*}\left(\left\{\varphi_{t}\right\}, \delta\right) \otimes_{\widehat{\Lambda}_{\omega, \eta, U}}\right. \\
& \left.\mathrm{F}\left(\widetilde{\Lambda}_{\omega, \eta}\right)\right) \\
= & \operatorname{dim}_{\mathrm{F}\left(\widehat{\Lambda}_{\omega, \eta, U}\right)} \mathrm{H}^{*}\left(\widehat{\operatorname{CFN}}_{U}^{*}\left(\left\{\varphi_{t}\right\}, \delta\right) \otimes_{\widehat{\Lambda}_{\omega, \eta, U}}\right. \\
& \left.\mathrm{F}\left(\widehat{\Lambda}_{\omega, \eta, U}\right)\right) \\
= & \operatorname{dim}_{\mathrm{F}\left(\widehat{\Lambda}_{\omega, \eta, U}\right)} \widehat{\operatorname{HFN}}_{U}^{*}\left(\left\{\varphi_{t}\right\}, J\right) \otimes_{\widehat{\Lambda}_{\omega, \eta, U}} \mathrm{~F}\left(\widehat{\Lambda}_{\omega, \eta, U}\right) \\
= & \operatorname{rank}_{\widehat{\Lambda}_{\omega, \eta, U}} \widehat{\operatorname{HFN}}_{U}^{*}\left(\left\{\varphi_{t}\right\}, J\right)
\end{aligned}
$$

Combining with Theorem 4.6, we get the following:

Proposition 4.7. $\operatorname{rank}_{\widetilde{\Lambda}_{\omega, \eta}} \operatorname{HFN}^{*}\left(\left\{\varphi_{t}\right\}, J ; \widetilde{M}\right)=\operatorname{rank}_{\widetilde{\Lambda}_{\omega, \eta+c}} \operatorname{HFN}^{*}\left(\left\{\phi_{t}^{\theta_{t}} \circ\right.\right.$ $\left.\left.\varphi_{t}\right\}, J ; \widetilde{M}\right)$.

Note that we do not claim that $\operatorname{rank}_{\Lambda_{\omega, \eta}} \operatorname{HFN}^{*}\left(\left\{\varphi_{t}\right\}, J\right)$ coincides with $\operatorname{rank}_{\Lambda_{\omega, \eta+c}} \operatorname{HFN}^{*}\left(\left\{\phi_{t}^{\theta_{t}} \circ \varphi_{t}\right\}, J\right)$. We define an equivalence relation on $V=$ $\operatorname{ker}\left\{\pi^{*}: \mathrm{H}^{1}(M ; \mathbf{R}) \rightarrow \mathrm{H}^{1}(\widetilde{M} ; \mathbf{R})\right\}$ by setting $\eta \sim \eta^{\prime}$ if and only if we have $\operatorname{rank}_{\widetilde{\Lambda}_{\omega, \eta}} \operatorname{HFN}^{*}\left(\left\{\varphi_{t}\right\}, J ; \widetilde{M}\right)=\operatorname{rank}_{\widetilde{\Lambda}_{\omega, \eta^{\prime}}} \operatorname{HFN}^{*}\left(\left\{\varphi_{t}^{\prime}\right\}, J: \widetilde{M}\right)$. Here $\eta\left(\right.$ resp. $\left.\eta^{\prime}\right)$ is the flux of $\left\{\varphi_{t}\right\}$ (resp. $\left\{\varphi_{t}^{\prime}\right\}$ ). Proposition 4.7 implies that $\left\{\eta \mid \eta \sim \eta_{0}\right\}$ is an open subset of $V$. Hence $V$ is decomposed into disjoint open subsets. Since $V$ is connected, all elements are equivalent. In other words, we obtained the following:

Proposition 4.8. $\operatorname{rank}_{\widetilde{\Lambda}_{\omega, \eta}} \operatorname{HFN}^{*}\left(\left\{\varphi_{t}\right\}, J ; \widetilde{M}\right)$ does not depend on $[\eta] \in V$.

If $[\eta] \in V$ is sufficiently small, we can compute the Floer-Novikov cohomology. Namely, for a sufficiently $C^{1}$-small Morse 1-form $\eta$, the FloerNovikov coboundary operator does not contain any quantum contribution, i.e., it is described by only bounded local-gradient flow lines. Hence the Floer-Novikov cohomology for such an $\eta$ is described by the Novikov cohomology for $\eta$, see $[\mathbf{5}, \mathbf{7}]$. We obtain the following: 
Theorem 4.9. Let $\eta$ be a sufficiently small element in $V$. If $\eta$ is in general position in the sense of Remark 4.2, we have

$$
\operatorname{rank}_{\widetilde{\Lambda}_{\omega, \eta}} \operatorname{HFN}^{*}\left(\left\{\varphi_{t}\right\}, J ; \widetilde{M}\right)=\sum_{p} \operatorname{rank}_{\Lambda_{\eta}} \operatorname{HN}^{p}(M,[\eta]) .
$$

In particular,

$$
\operatorname{rank}_{\widetilde{\Lambda}_{\omega, \eta}} \operatorname{HFN}^{*}\left(\left\{\varphi_{t}\right\}, J ; \widetilde{M}\right) \geq \sum_{p} \min -\operatorname{nov}_{p}(M) .
$$

Proof. When $\eta \in V$ is in general position in the sense of Remark 4.2, the cochain complex $\left(\mathrm{CFN}^{*}\left(\left\{\phi_{t}\right\}, J ; \widetilde{M}\right), \delta\right)$ coincides with the usual FloerNovikov cochain complex $\left(\mathrm{CFN}^{*}\left(\left\{\phi_{t}\right\}, J\right), \delta\right)$. Moreover, as we have seen in $[\mathbf{5}, \boldsymbol{7}]$, the bounded local gradient flow lines are the only connecting orbits contributing to the Floer-Novikov coboundary operator $\delta$. Hence we obtain the first equality. The second inequality is clear by the definition of the minimal Novikov number.

\section{Comparison result}

In the previous section, we established an estimate for the rank of the cohomology $\operatorname{HFN}^{*}\left(\left\{\varphi_{t}\right\}, J ; \widetilde{M}\right)$. In this section, we give an estimate for the rank of $\operatorname{HFN}^{*}\left(\left\{\varphi_{t}\right\}, J\right)$. Here $\pi: \widetilde{M} \rightarrow M$ is a covering space so that $\pi^{*} \eta$ is exact. Note that we use a smaller covering space of the loop space of $M$ in order to define the Floer-Novikov cochain complexes $\left(\operatorname{CFN}^{*}\left(\left\{\varphi_{t}\right\}, J\right), \delta\right)$ than in the definition of $\left(\mathrm{CFN}^{*}\left(\left\{\varphi_{t}\right\}, J ; \widetilde{M}\right), \delta\right)$. Namely, $\widetilde{\mathcal{L}} M$ in Section 3 is the quotient of $\widetilde{\mathcal{L}} \widetilde{M}$ by the group generated by $(g, h) \in \pi_{1}(M) \times \pi_{2}(M)$ such that $I_{\eta}(g)+\mathcal{I}_{\omega}(h)=0$ and $c_{1}(M)[h]=0$. We consider the Floer-Novikov complex $\left(\widehat{\mathrm{CFN}}_{U}^{*}\left(\left\{\varphi_{t}\right\}, J ; \widetilde{M}\right), \delta\right)$ over $\widehat{\Lambda}_{\omega, \eta, U}$. We show that the degree zero part of $\widehat{\Lambda}_{\omega, \eta, U}$ is a principal ideal domain.

Lemma 5.1. $\widehat{\Lambda}_{\omega, \eta, U}^{0}$ is a principal ideal domain.

Proof. Pick $\theta \in U$ in a general position so that the function $\mathcal{I}_{\omega}+\mathcal{I}_{\eta+\theta}$ is injective. Then the conclusion is proved by the standard argument (see e.g., Section $4[4])$.

Now we prove the following theorem comparing the Floer-Novikov cohomology associated to the covering $\widetilde{M} \rightarrow M$ and the genuine Floer-Novikov cohomology.

Theorem 5.2. $\operatorname{rank}_{\widetilde{\Lambda}_{\omega, \eta}} \operatorname{HFN}^{*}\left(\left\{\varphi_{t}\right\}, J ; \widetilde{M}\right)=\operatorname{rank}_{\widehat{\Lambda}_{\omega, \eta, U}} \widehat{\operatorname{HFN}}_{U}^{*}\left(\left\{\varphi_{t}\right\}\right) \leq$ $\operatorname{rank}_{\Lambda_{\omega, \eta}} \operatorname{HFN}^{*}\left(\left\{\varphi_{t}\right\}\right)$.

Proof. The first equality has been proved just before Proposition 4.7. We prove the second inequality. 
The variant $\widehat{\mathrm{HFN}}_{U}^{*}\left(\left\{\varphi_{t}\right\}\right)$ of the Floer-Novikov cohomology is a module over $\Lambda_{\omega, \eta, U}$ while the genuine Floer-Novikov cohomology $\operatorname{HFN}^{*}\left(\left\{\varphi_{t}\right\}\right)$ is a module over $\Lambda_{\omega, \eta}$. As for the coefficient rings, there is a natural homomorphism $\widehat{\Lambda}_{\omega, \eta, U} \rightarrow \Lambda_{\omega, \eta}$, the restriction of which to the degree zero part is $\widehat{\Lambda}_{\omega, \eta, U}^{0} \rightarrow \Lambda_{\omega, \eta}^{0}$.

Let $\widehat{\mathrm{ZFN}}_{U}^{p}$ and $\widehat{\mathrm{BFN}}_{U}^{p}$ be the submodules of cocycles and coboundaries of $\left(\widehat{\mathrm{CFN}}_{U}^{*}\left(\left\{\varphi_{t}\right\}, J ; \widetilde{M}\right), \delta\right)$, respectively. Denote by $\mathrm{ZFN}^{p}$ and $\mathrm{BFN}^{p}$ the submodules of cocycles and coboundaries in $\operatorname{CFN}^{p}\left(\left\{\varphi_{t}\right\}, J\right)$, respectively. Then we have

$$
\begin{gathered}
\widehat{\operatorname{BFN}}_{U}^{p} \otimes_{\widehat{\Lambda}_{\omega, \eta, U}^{0}} \Lambda_{\omega, \eta}^{0}=\mathrm{BFN}^{p}, \\
\widehat{\mathrm{ZFN}}_{U}^{p} \otimes_{\widehat{\Lambda}_{\omega, \eta, U}^{0}}^{0} \Lambda_{\omega, \eta}^{0} \subset \mathrm{ZFN}^{p} .
\end{gathered}
$$

Hence, we have

$$
\widehat{\operatorname{HFN}}_{U}^{p}\left(\left\{\varphi_{t}\right\}, J ; \widetilde{M}\right) \otimes_{\widehat{\Lambda}_{\omega, \eta, U}^{0}} \Lambda_{\omega, \eta}^{0} \subset \operatorname{HFN}^{p}\left(\left\{\varphi_{t}\right\}, J\right) .
$$

Since $\widehat{\mathrm{CFN}}_{U}^{*}\left(\left\{\varphi_{t}\right\}, J ; \widetilde{M}\right)$ is finitely generated over the principal ideal domain $\widehat{\Lambda}_{\omega, \eta, U}^{0}$, there is an isomorphism

$$
\widehat{\operatorname{HFN}}_{U}^{p}\left(\left\{\varphi_{t}\right\}, J ; \widetilde{M}\right) \cong\left(\widehat{\Lambda}_{\omega, \eta, U}^{0}\right)^{\oplus l_{p}} \oplus \text { Tors, }
$$

where Tors is a torsion module over $\widehat{\Lambda}_{\omega, \eta, U}^{0}$. Therefore, $\widehat{\operatorname{HFN}}_{U}^{p}\left(\left\{\varphi_{t}\right\}, J ; \widetilde{M}\right)$ $\otimes_{\widehat{\Lambda}_{\omega, \eta, U}^{0}} \Lambda_{\omega, \eta}^{0}$ contains a free module of rank $l_{p}$ over $\Lambda_{\omega, \eta}^{0}$, which is also a principal ideal domain. (In fact, the degree zero part $\Lambda_{\omega, \eta}^{0}$ of the Novikov ring with coefficients in a field is also a field.) Thus we find that

$$
\operatorname{rank}_{\widehat{\Lambda}_{\omega, \eta, U}^{0}} \widehat{\operatorname{HFN}}_{U}^{p}\left(\left\{\varphi_{t}\right\}, J ; \widetilde{M}\right) \leq \operatorname{rank}_{\Lambda_{\omega, \eta}^{0}} \operatorname{HFN}^{p}\left(\left\{\varphi_{t}\right\}, J\right) .
$$

Note also that, if the minimal Chern number $N$ of $(M, \omega)$ is not zero,

$$
\operatorname{rank}_{\Lambda_{\omega, \eta}} \operatorname{HFN}^{*}\left(\left\{\varphi_{t}\right\}, J\right)=\sum_{p=0}^{2 N-1} \operatorname{rank}_{\Lambda_{\omega, \eta}^{0}} \operatorname{HFN}^{p}\left(\left\{\varphi_{t}\right\}, J\right)
$$

and

$$
\operatorname{rank}_{\widehat{\Lambda}_{\omega, \eta, U}} \widehat{\operatorname{HFN}}_{U}^{*}\left(\left\{\varphi_{t}\right\}, J ; \widetilde{M}\right)=\sum_{p=0}^{2 N-1} \operatorname{rank}_{\widehat{\Lambda}_{\omega, \eta, U}^{0}} \widehat{\operatorname{HFN}}_{U}^{p}\left(\left\{\varphi_{t}\right\}, J ; \widetilde{M}\right) .
$$

If $N=0, \Lambda_{\omega, \eta}$ and $\Lambda_{\omega, \eta, U}$ coincide with their degree zero part $\Lambda_{\omega, \eta}^{0}$ and $\Lambda_{\omega, \eta, U}^{0}$, respectively, and we find that

$$
\operatorname{rank}_{\Lambda_{\omega, \eta}} \operatorname{HFN}^{*}\left(\left\{\varphi_{t}\right\}, J\right)=\sum_{p} \operatorname{rank}_{\Lambda_{\omega, \eta}^{0}} \operatorname{HFN}^{p}\left(\left\{\varphi_{t}\right\}, J\right)
$$


and

$$
\operatorname{rank}_{\widehat{\Lambda}_{\omega, \eta, U}} \widehat{\operatorname{HFN}}_{U}^{*}\left(\left\{\varphi_{t}\right\}, J ; \widetilde{M}\right)=\sum_{p} \operatorname{rank}_{\widehat{\Lambda}_{\omega, \eta, U}^{0}} \widehat{\operatorname{HFN}}_{U}^{p}\left(\left\{\varphi_{t}\right\}, J ; \widetilde{M}\right) .
$$

From these facts, we obtain the second inequality.

Combined with Proposition 4.8 and Theorem 4.9, we obtain the following theorem, which is the goal of this section.

Theorem 5.3. $\operatorname{rank}_{\Lambda_{\omega, \eta}} \operatorname{HFN}^{*}\left(\left\{\varphi_{t}\right\}\right) \geq \sum_{p} \min -\operatorname{nov}_{p}(M)$.

Proof. By Proposition 4.8 and Theorem 4.9, we find that

$$
\operatorname{rank}_{\widetilde{\Lambda}_{\omega, \eta}} \operatorname{HFN}^{*}\left(\left\{\varphi_{t}\right\}, J ; \widetilde{M}\right) \geq \sum_{p} \min -\operatorname{nov}_{p}(M) .
$$

Combining this inequality with Theorem 5.2, we obtain the conclusion.

Theorem 1.1 is a direct consequence of Theorem 5.3.

\section{References}

[1] A. Floer, Symplectic fixed points and holomorphic spheres, Comm. Math. Phys. 120 (1989), 575-611.

[2] K. Fukaya, Y.-G. Oh, H. Ohta and K. Ono, Lagrangian intersection Floer theory obstruction and anomaly, preprint.

[3] K. Fukaya and K. Ono, Arnold conjecture and Gromov-Witten invariants, Topology 38 (1999), 933-1048.

[4] H. Hofer and D. Salamon, Floer homology and Novikov rings, in 'The Floer Memorial volume' (H. Hofer, C.H. Taubes, A. Weinstein and E. Zehnder, eds.), Progress in Math. 133, Birkhäuser, 1995, 483-524.

[5] H.-V. Lê and K. Ono, Symplectic fixed points, the Calabi invariant and Novikov homology, Topology 34 (1995), 155-176.

[6] G. Liu and G. Tian, Floer homology and Arnold conjecture, J. Diff. Geom., 49 (1998), $1-74$.

[7] K. Ono, Floer-Novikov cohomology and the flux conjecture, preprint, 2004 (Revised October 2005).

[8] P. Seidel, Braids and symplectic four-manifolds with abelian fundamental group, preprint, arXiv: math.SG/0202135.

Department of Mathematics

HOKKAIDO UNIVERSITY

SAPPORO 060-0810, JAPAN

E-mail address: ono@math.sci.hokudai.ac.jp

Received 01/21/2005, revised 11/02/2005. Partly supported by the Grant-in-Aid for Scientific Research no. 14003419, Japan Society for the Promotion of Sciences. 
